\title{
Strates
}

STRATES Matériaux pour la recherche en sciences sociales

$11 \mid 2004$

Jeune recherche, la vitalité d'un laboratoire

\section{Barcelone par ses paysages contée : pertinence du dialogue entre romans et récits d'habitants}

Sophie Savary

\section{(2) OpenEdition \\ 12 Journals}

Édition électronique

URL : http://journals.openedition.org/strates/420

ISSN : $1777-5442$

Éditeur

Laboratoire Ladyss

Édition imprimée

Date de publication : 1 janvier 2004

ISSN : 0768-8067

\section{Référence électronique}

Sophie Savary, «Barcelone par ses paysages contée : pertinence du dialogue entre romans et récits d'habitants ", Strates [En ligne], 11 | 2004, mis en ligne le 14 janvier 2005, consulté le 07 septembre 2020. URL : http://journals.openedition.org/strates/420

Ce document a été généré automatiquement le 7 septembre 2020

Tous droits réservés 


\title{
Barcelone par ses paysages contée : pertinence du dialogue entre romans et récits d'habitants
}

\author{
Sophie Savary
}

1 L'origine de ma recherche sur Barcelone s'enracine dans deux amours qui échappent à la seule géographie : la ville et Barcelone. La ville pour sa rudesse mêlée de chaleur, «l'aliment préféré des chiens » selon le mot de Daniel Pennac, généreuse pour les sans nom, sans lieu, sans lien. Et Barcelone parce que dans la grande famille des villes que j'habite, ou qui m'habite, Barcelone s'est imposée peu à peu, sans doute pour m'être frottée des centaines d'heures à ses murs et m'être rafraîchie dans sa mer toute neuve. La relation entre la ville, ou certains lieux de la ville, et moi est celle de l'empathie, qui s'est tissée au fur et à mesure que s'accumulaient les expériences paysagères, les souvenirs, les réflexions et la connaissance sur les lieux.

2 Tout un chacun peut vivre cette relation avec son environnement, mais l'on se transforme en géographe lorsque naît le désir de lui donner du sens ou des sens, de la rendre lisible et de l'étendre à l'échelle de la société. Dresser une géographie de la ville et de ses lieux consiste alors à rendre compte du sens de la relation habitants / environnement, ce que l'on peut désigner par le terme d'urbanité. Son appréhension suppose de retenir à la fois sa dimension sensible (mélange de sensation et d'affectivité), son ordonnancement, ses logiques, ses orientations.

Pour cela, il faut puiser dans les ressources infinies et insuffisamment exploitées de l'imaginaire, exprimé le plus clairement sinon le plus «naturellement » par le langage. N'est-ce pas, dans ce cas, faire appel à des experts que d'écouter les écrivains de fiction? Ils consacrent leur travail, et parfois leur existence, à ordonner le monde en imaginant des fictions, en cherchant à rendre une réalité par le langage. Mon objectif ici est de montrer que la richesse de ces textes ne pourra cependant être atteinte que si on les fait dialoguer avec les discours d'habitants non experts, ordinaires ${ }^{1}$. Il s'agit donc d'une recherche fondée sur les métissages discursifs (textes littéraires / paroles 
d'habitants) et disciplinaires (Littérature / Géographie) qui, grâce à l'identification, la définition, l'analyse de paysages tente de construire le sens d'une ville : Barcelone.

Quatre écrivains pour une ville

4 Reconstruire l'urbanité d'une ville suppose le questionnement suivant : comment réel et imaginaire se combinent-ils pour construire l'identité de la ville et de ses habitants? Comment histoire et mythologie, monde adulte et monde de l'enfance, exigences matérielles et rêves fusionnent-ils pour construire cette identité urbaine?

5 Détecter les indices d'urbanité n'est possible qu'en repérant la façon dont ils s'expriment: les paysages sont ces traces de relation habitants / ville et constituent une de ses principales formes d'expression. Ils n'apparaissent que sous forme de représentations individuelles ou collectives qui cristallisent l'identité de chacun et des groupes. L'expression artistique est particulièrement appropriée pour saisir cette urbanité entre réel et imaginaire, pour définir cette réalité abstraite : la réalité telle que la conçoit Proust à partir de l'élaboration de l'œuvre d'art ${ }^{2}$ est bien celle à laquelle je voulais accéder. Cette réalité prend forme dans le langage ${ }^{3}$ qui joue donc un rôle fondateur dans la réalisation et l'organisation paysagère ; les discours s'imposent ainsi comme mode d'approche privilégié des paysages. Les discours littéraires, de par leur statut d'œuvre d'art, permettent de rendre compte de la dimension à la fois sensible et rationnelle des paysages, de leur caractère individuel chargé d'une dimension collective. Ce sont donc à la fois des outils et des gisements de matériaux mobilisables pour la réalisation d'une géographie que l'on pourrait qualifier de sensible ${ }^{4}$.

6 Mis à part mon attachement à la ville de Barcelone et l'intuition qu'elle "parlerait " plus que tout autre, celle-ci était toute désignée pour accomplir la tâche entreprise. Soumise à de grands bouleversements paysagers (transformations des années 80 en lien ou non avec les Jeux Olympiques de 1992, projets urbanistiques fondant la légitimité de l'événement Forum des cultures en 2004, etc.), la capitale de la Catalogne s'inscrit dans une longue tradition urbanistique et architecturale. L'innovation en ce domaine y est unique et cela génère chez ses habitants une fierté à la hauteur des efforts entrepris. Pour cette raison, et bien d'autres, les Barcelonais vouent un véritable culte à leur ville ; elle offre donc un intérêt tout particulier pour analyser et interpréter la relation affective, symbolique ou matérielle qu'ils entretiennent avec elle.

7 L'originalité de l'histoire urbanistique et l'amour des habitants pour leur ville ne suffiraient cependant pas à justifier son élection pour un travail fondé sur l'analyse de romans. C'est bien son caractère éminemment littéraire qui la rend attractive et qui l'impose comme protagoniste de l'étude. Barcelona, ciudad de novela, Barcelone, ville de roman, c'est ainsi qu'Isabel de Villalonga intitulait son article de La Vanguardia du 2 août 2001: comme des dizaines de cycles de conférences, d'articles ou d'ouvrages, la journaliste pointait du doigt le caractère littéraire de la ville et le poids de la littérature dans la construction de l'identité barcelonaise ${ }^{5}$; ses écrivains entretiennent avec elle une relation quasi-existentielle.

8 J'ai choisi parmi eux quatre auteurs contemporains pour qui Barcelone, ou certains de ses quartiers, forment la chair de leurs romans : Luis Goytisolo (1935) est un auteur influent en Catalogne bien que peu lu ${ }^{6}$; Juan Marsé (1933), lauréat 2001 du plus grand prix littéraire espagnol, le Premio Nacional de Narrativa, déjà détenteur d'une dizaine de prix nationaux et internationaux, est le représentant du quartier Gracia-Guinardó ; Eduardo Mendoza (1943) a dédié son œuvre à Barcelone : La ciudad de los prodigios (1986) l'a véritablement consacré dans son pays et dans le monde ${ }^{7}$; Manuel Vázquez 
Montalbán (1939) est sans doute l'auteur qui diffuse le plus ses idées et ses représentations du fait de la diversité de son activité (romans, poésie, essais, enseignement universitaire, chroniqueur journalistique) et de sa reconnaissance auprès du public et des cercles littéraires.

Ces habitants amoureux de leur ville se font un devoir d'être des décrypteurs / transcripteurs du monde qui les entoure, et cela d'autant plus qu'ils ont en parallèle une activité de chroniqueur journalistique ${ }^{8}$ (ils s'exercent donc à l'art de la fiction et à la critique raisonnée du monde environnant) et qu'ils représentent la génération dite posguerra $a^{9}$. Leur préoccupation principale est la récupération de la mémoire, celle des temps traumatiques de la période franquiste que les cercles d'élites et de puissants démocrates ont voulu effacer lors de la "transition ", et qui correspond à leur enfance (Cf. surtout Goytisolo, Marsé et Montalbán); celle aussi des origines, qui s'ancre pour Mendoza dans la période 1880-1930. Leur quête du sens de leur monde passe ainsi par l'écoute et l'expression de leurs expériences personnelles, leurs émotions et obsessions, mais pas seulement : ils se documentent aussi très précisément à partir d'archives, de lectures de spécialistes urbanistiques ou d'histoires de l'art ${ }^{10}$. Juan Marsé, quant à lui, construit ses histoires, ses descriptions, ses dialogues à partir d'images qu'il a en mémoire et qui servent de tremplin à l'écriture: c'est à partir de l'observation remémorée que la fiction naît, procédé qui n'est pas sans rappeler la méthode proustienne fondée sur le souvenir que l'écriture réactive selon une formule où se mêlent l'imagination, la sensibilité et l'intelligence.

10 Je lis donc leur roman avec légèreté, afin de réaliser mon propre travail d'imagination sur les textes, et j'opère des analyses rigoureuses et précises selon des méthodes et des modes d'approche littéraires; je propose en parallèle une interprétation des textes à partir de mesures, relevés et classements (par exemple relevé, comptabilisation, classement des composants paysagers de toute nature); en les rapprochant avec des documents extra-littéraires; en réalisant une cartographie interprétative des mondes dévoilés. Le sens des textes est donc le produit d'un dialogue entre expérience littéraire et géographique ${ }^{11}$.

Créer une géographie métisse : générer un dialogue

entre habitants littéraires, ordinaires, expertsPourquoi l'initier par les textes

littéraires?

Démontrer la nécessité du dialogue entre ces deux langages pour élaborer une géographie plus "sensible", suppose de remonter à la source de l'intérêt porté aux discours littéraires. Il faut tout d'abord convaincre de la richesse spécifique produite par la littérature pour la société, ce qui exige de se confronter à deux questions majeures : les paysages des romans étudiés proposent-ils de nouvelles interprétations du sens de la ville ou reposent-ils sur une représentation collective dont les auteurs rendraient compte ? Quelle place attribuer à la dimension collective et quelle place à la dimension individuelle dans le processus de création paysagère?

Pierre Lassave (2002) résume ainsi les trois exploitations possibles et reconnues de la littérature par les sciences sociales : « un corpus de données, une ressource cognitive et un modèle d'énonciation » (p. 37). La valeur documentaire, des romans notamment, n'est pourtant pas ce qui rend innovante la littérature dans son approche du monde. En tant que "ressources cognitives » ou «stimulant cognitif indirect» (Lassave, 2002, p. 38), elle fournit davantage à la société des schèmes de compréhension et de perception du monde, qui constituent le terreau de représentations collectives. Ainsi en est-il de 
l'invention du terme et du mythe du barrio chino à Barcelone par la littérature : ce quartier qui chevauche la partie méridionale des Ramblas, rassemblait dans les années 50 toutes les composantes du quartier de bas-fonds urbain (prostitution, amusement, délabrement, charme de la décadence). Un roman de Francesc Madrid, relayé par des romans français, a dénommé un espace qui n'a jamais eu un quelconque caractère chinois, et amplifié les caractéristiques de perdition au point que ce fut la seule image du quartier qui traversât ses frontières, attirant ainsi les jeunes bourgeois barcelonais qui aimaient se mêler à la bohême ici installée. Aujourd'hui, malgré la réhabilitation cannibale de cet espace, les touristes continuent à chercher dans le barrio une atmosphère canaille qui n'existe quasiment plus et Montalbán, natif de ce quartier, comme les autres auteurs perpétuent et contribuent à distordre le mythe. Au-delà de l'élaboration d'images ou de réputation, les romans nous offrent d'autres formes de "ressources cognitives». C'est ainsi qu'en identifiant les signes, les motifs paysagers, les configurations récurrents chez un auteur, ou dans plusieurs œuvres, j'ai senti que j'approchais du sens de certains quartiers et par là même que je me dirigeais doucement vers la composition de l'urbanité de l'entité monstrueuse de la ville tout entière ${ }^{12}$.

La littérature proposerait aussi des "modèles d'énonciation"; le terme est un peu restrictif pour désigner ce qu'apporte la littérarité d'un texte, à savoir des propositions de formulations du monde à valeur heuristique : en montrant ce qui n'a jamais été montré, car jamais verbalisé ainsi, en mettant des mots sur des expériences vécues, même fictives. La vision du monde présente dans le style est une proposition de lecture du réel, à la fois subjective (car individuelle) et sociale (l'auteur et ses productions s'inscrivent toujours dans un ensemble de codes sociaux, en particulier de codes langagiers internes et externes à la littérature). Par ce travail de reformulation de la réalité, le texte littéraire permet de mieux comprendre les procédés discursifs qui créent des paysages.

Il faut ajouter aux trois catégories proposées par Lassave, les interprétations théoriques que développent les auteurs dans leurs romans en matière de sociologie, d'urbanisme, de politique, d'histoire, etc. Les textes littéraires n'offrent pas qu'une interprétation poétique du monde, ils ouvrent des pistes de réflexion que les spécialistes n'ont pas toujours explorées ou abordées selon des points de vue différents. Ainsi en est-il de l'analyse sociale de Barcelone dressée par Luis Goytisolo dans son roman Recuento (1973) et dans les autres volumes de sa tétralogie Antagonía. Elle est originale tout en étant une réinterprétation de la méthode proustienne de lecture et de compte-rendu de la société. Vázquez Montalbán de son côté travaille particulièrement la question de l'occultation de la mémoire pendant la période de transición et la prolongation du processus aujourd'hui par les actions politico-urbanistiques menées sur Barcelone.

Toutes ces propriétés que recèle la littérature ne peuvent occulter cependant ce qui est peut-être le plus intéressant: le recours à la fiction. L'invention, les artifices de la fiction, bien loin d'introduire un biais négatif dans la saisie et la compréhension des paysages d'un roman, procurent des sources de connaissances uniques. Quelques exemples suffisent pour convaincre de la richesse de la narration fictionnelle. Ainsi Juan Marsé, pour rendre compte d'un moment ou d'une durée, exploite-t-il la fonction temporelle des paysages ${ }^{13}$ : cet artifice est une richesse heuristique (il nous montre un paysage particulier) et théorique (il fixe notre attention sur la dimension temporelle de l'expérience paysagère et de sa représentation, aspect auquel prêtent peu attention les 
géographes). Autre instrument intéressant de la fiction : le personnage. Il constitue une source de connaissances pour les sciences sociales et particulièrement sur les paysages. La relation personnage / paysage construite au sein d'un roman révèle le fonctionnement de la relation homme / paysage dans le monde référentiel. Dans tous les romans étudiés, et en particulier dans ceux de Marsé et de Montalbán, on a pu analyser comment certains personnages sont construits par les paysages qu'ils perçoivent et qu'ils expriment, soit au style direct, soit par l'intermédiaire d'un narrateur ${ }^{14}$.

Grâce à leur art de la narration et de l'intrigue, grâce à leurs formulations originales et créatrices de sens, les romanciers nous conditionnent pour comprendre le monde de l'intérieur, nous placent en situation d'immersion dans un monde où imaginaire et réel sont effectivement mêlés. Ils nous aident à l'appréhender et à en rendre compte d'une manière à la fois sensible, imaginative et rationnelle, nous ouvrant ainsi à une géographie véritablement duelle, où la problématique de la tension entre réel et imaginaire, qui rencontre celle de la vérité et de la fiction, peut être reformulée. Nous sommes alors au cœur de ce que peut signifier élaborer une autre pratique géographique. Pour nous persuader de la pertinence de la littérature pour comprendre comment l'on peut reformuler cette problématique, considérons le statut du référent dans le roman. Le référent ${ }^{15}$ est ce qui émerge du réel dans la fiction et par conséquent ce qui dans la fiction permet de recréer un monde vraisemblable. Le " monde » d'un roman est construit par emboîtement d'éléments réels « objectifs » reconstruits par l'écriture (ex. : les Expositions Universelles de Barcelone décrites par Mendoza dans La ciudad...) et par des éléments issus du seul monde de l'auteur, qui relèvent du référent subjectif (ses images, son histoire, ses fantasmes, son éducation, ses amis et rencontres) toujours enchâssé dans une réalité sociale réelle. Les descriptions de paysage constituent des exemples paradigmatiques pour saisir ce phénomène d'enchevêtrement entre réel et imaginaire : les descriptions, type tableaux composés selon des canons picturaux ou cinématographiques, sont structurées par des codes génériques qui modèlent la syntaxe, le lexique, les thématiques (ils sont donc constitués d'artifices) et s'ancrent généralement dans le réel par des toponymes, des itinéraires géographiques, des connecteurs de lieu, etc. ${ }^{16}$ Les descriptions paysagères ont aussi souvent recours à la fois à des procédés réalistes et fantastiques, ou réalistes et burlesques, ou encore entièrement surréels pour faire surgir une image du monde qui est celle interprétée par l'auteur.

17 On pourrait croire à l'issue de cet aperçu des richesses qu'offre la littérature pour étudier les paysages qu'il serait bien inutile d'aller chercher ailleurs. Pourquoi demeure néanmoins cette réticence à se cantonner aux discours littéraires? En quoi les croiser avec des discours d'une autre nature serait-il plus pertinent?

Textes littéraires, discours d'experts, récits d'habitants : le dialogue nécessaire

18 Ce qui nous empêche, en tant que géographe, de demeurer dans la chaleur humaine de la littérature n'est sans doute dans un premier temps qu'un réflexe de conformité à l'institution universitaire et aux pétitions de principe de la discipline qui clame la scientificité de la géographie. Si la géographie est une science humaine ou science sociale, elle relève du paradigme scientifique; ses travaux doivent donc pour être valides respecter des règles imprescriptibles comme les principes d'objectivité de la connaissance ou de non-contradiction et fonder son raisonnement sur une expérimentation à partir de laquelle des résultats pourront être affirmés selon le 
principe de l'administration de la preuve. La littérature de son côté aurait un rôle à jouer dans la connaissance des hommes, mais son apport se cantonnerait dans le domaine subjectif : elle explorerait les domaines de l'imaginaire, des sentiments, des passions. Cette distinction entre science et littérature paraît bien éculée dans l'état des débats sur la question aujourd'hui ${ }^{17}$ et les nombreux travaux en géographie de grande qualité, fondés uniquement sur un matériau littéraire, ont sorti la géographie de l'ornière dans laquelle elle s'était enlisée de ne se croire qu'une science et non avant tout une humanité.

19 La réticence disciplinaire surmontée, l'intérêt du dialogue entre textes littéraires et autres formes de discours demeure. En premier lieu, il est opportun de rappeler à quel point confronter des discours de spécialistes, des discours littéraires (et artistiques en général) et des discours vernaculaires, permet de renouveler la réflexion concernant les notions transdisciplinaires. Je prendrai pour exemple la notion de méditerranéité. La pertinence de la notion et ses critères de définition ont été retravaillés récemment dans un ouvrage collectif sur la Méditerranée latine : les auteurs veulent dans leur introduction déterminer des facteurs de définition de cet espace. Ils ont recours notamment à une enquête réalisée en $1995^{18}$ dont les résultats intriguent: le paysage n'est considéré comme facteur d'identification de l'arc latin que pour $8,6 \%$ des personnes interrogées, bien après le facteur linguistique (langues latines), la proximité géographique, le caractère dynamique de ces régions, la tradition commerciale et la culture catholique. Cette enquête et les développements suivants concernant les paysages méditerranéens confirment le carcan restrictif dans lequel est cantonnée la notion de paysage d'une part et celle de méditerranéité d'autre part. Pour en sortir, il faut aborder la question selon un tout autre point de vue, à partir d'autres discours ${ }^{19}$. Cela peut consister à chercher à quoi servent, dans la fiction, les éléments caractéristiques du monde méditerranéen (qu'ils soient explicitement qualifiés de méditerranéens ou associées au monde méditerranéen dans le texte, ou que cette qualification soit réalisée logiquement par le lecteur). La quête de leur sens est ici réalisée à tous les niveaux : au niveau de la narration, du fonctionnement de l'intrigue, dans la construction du monde, de l'axiologie du roman, de sa poétique, de sa signification métaphorique ou symbolique. L'on peut aussi traquer les marqueurs de méditerranéité et repérer si l'on en découvre de nouveaux, voir jusqu'où s'étend leur sens, en particulier dans la sphère symbolique et imaginaire.

Concrétisons ce qui vient d'être formulé par un exemple issu de l'étude de l'œuvre de Juan Marsé. Les marqueurs de méditerranéité dans ses romans sont nombreux et se combinent pour construire un monde qui est à la fois référentiel et personnel, contenu tout entier dans un quartier que l'on a coutume de nommer son barrio mental. Parmi ces marqueurs, j'ai repéré une composante paysagère qui me semble enrichir notre approche de la méditerranéité : le leitmotiv de la poussière rouge. En reconstruisant la généalogie de cette composante, j'ai constaté qu'elle était présente dès son premier "grand roman ", Últimas tardes con Teresa, et qu'elle s'est imposée peu à peu jusqu'à devenir, dans son dernier roman Rabos de lagartija, un marqueur territorial fondamental. Une des descriptions de ce roman reconstitue quasiment l'intégralité de l'histoire de l'élaboration de cette image paysagère obsessionnelle ${ }^{20}$. Le premier élément de la description rappelle l'origine de cette poussière rouge : "esta tierra arcillosa y encrestada " ("cette terre argileuse et altière ») complétée un peu plus loin par l'élément "grietas rojizas » (« crevasses rougeâtres ») puis apparaît le fruit de cette topographie et de ce sol particulier « una efusión rojiza de polvo, como el resplandor de un 
incendio » (" une effusion de poussière rougeâtre, comme l'éclat d'un incendie »). Fidèle à l'histoire de son élaboration dans l'œuvre, l'image est d'abord née de l'observation d'un fait géographique: la présence d'une terre argileuse de couleur rouge (on reconnaît la terra rossa méditerranéenne), associée le plus souvent, mais pas ici, aux autres caractéristiques de l'aridité et du vent. Dans d'autres passages, et cela depuis Ronda, une autre observation d'ordre socio-géographique explique la transformation en poussière de cette terre à savoir que les rues ne sont pas toujours goudronnées dans les années 40 dans ce quartier et qu'il y a de nombreux terrains vagues ou espaces abandonnés. C'est donc à la fois la connaissance d'un environnement particulier (issu du monde référentiel et qui relève de la sphère réaliste de la construction du barrio mental) et la logique qui ont permis de détecter et d'élaborer un marqueur territorial d'ordre paysager connotant le caractère méditerranéen. Mais son élaboration ne s'arrête pas à cette sphère référentielle, réaliste, dans Rabos de Lagartija. Le sens y est étendu au domaine symbolique et imaginaire grâce à une pénétration progressive dans une atmosphère fantastique: la polysémie du terme grietas (à la fois fissures topographiques et estafilades sur un corps blessé, qui résonne dans le roman avec la blessure d'un personnage qui perd son sang dans le ravin), nous offre un sens instable qui nous propose de passer le seuil d'un monde où le sens n'est plus assuré par le raisonnement, mais par glissement métaphorique. Le dernier élément « efusión rojiza de polvo... » nous confirme que nous sommes entrés dans le monde fantastique, proposant d'associer à cette poussière un axe symbolique igné. La composition de cette description et le choix de son lexique nous permettent donc d'avoir accès, par degrés, aux deux côtés du miroir : le côté réaliste et le côté fantastique, qui sont indissociables pour pénétrer la réalité de cette composante paysagère devenu symbole de ce territoire.

21 De cet examen, rapidement résumé, de la poussière rouge dans les romans de Marsé, deux enseignements concernant le concept de méditerranéité peuvent être tirés. En premier lieu, la poussière rouge est un critère de reconnaissance du caractère méditerranéen d'un milieu et cela en particulier en ville (son origine édaphique et climatique, notamment, explicitée par Marsé, ainsi que sa combinaison avec d'autres marqueurs d'ordre topographique, climatique, édaphique, végétal, etc. présents dans les romans, prouvent que le monde méditerranéen est bien connoté dans cette composante paysagère). Le point de vue des habitants apporterait un élément supplémentaire de réflexion: la retrouve-t-on dans leurs discours comme marqueur identifiant du lieu et qu'en disent-ils ? Est-ce pour eux un attribut méditerranéen? En second lieu, le sens de ce marqueur méditerranéen est considérablement enrichi, notamment dans sa sphère symbolique, métaphorique et imaginaire par l'art de l'écrivain (et bien évidemment, seule une petite partie de ces sens a été rendue cidessus). C'est bien un motif identifiant à part entière que Marsé a reconstruit dans ses romans puisqu'il nous en propose un sens à la fois réel et imaginaire, rationnel et irrationnel.

22 Au-delà de la rencontre entre discours de spécialistes et discours littéraires, l'ultime argument qui l'emporte sur tout autre et qui les englobe pour déclarer nécessaire le dialogue composé de langages différents, est celui qui affirme que tout discours relève du phénomène d'intertextualité pris au sens large, c'est-à-dire celui qui rejoint la notion de dialogisme chez Bakhtine ${ }^{21}$ : cette conception ouvre les textes littéraires sur le monde social et admet que le texte est imprégné de langage ordinaire. Pour Bakhtine, le roman est le genre dialogique par excellence : la polyphonie est le fait de la 
plupart des romans modernes, y compris dans les monologues, et nos auteurs ne dérogent pas à cette règle. On comprend aisément dans ces conditions combien l'étude de discours ordinaires peut enrichir la lecture des romans et d'autant plus lorsqu'ils relèvent du genre réaliste ou du polar. Les auteurs sont dans le monde, ils connaissent les gens de la rue, sont parfois eux-mêmes issus de milieu populaire bien souvent urbain. On retrouve dans leur travail d'écriture une quête du vraisemblable et un désir de conserver la mémoire des «sans-paroles" en les décrivant, eux et leur environnement, leur langage, leurs manières d'être en société. Que ce soit les habitants marginaux et rebelles de Gracia-la Salud dans les années d'après-guerre réanimés par Marsé, ceux du Raval que Montalbán décrit ou les étudiants bourgeois des années 50 aux attitudes et langage faussement populaires démasqués par Goytisolo, tous n'acquièrent leur vraisemblance que grâce au travail effectué par les auteurs sur leur langue. Il est intéressant pour nous d'analyser les formules, les lexiques mobilisés pour réaliser leurs paysages ainsi que leurs modes d'émergence dans le discours. Est ce original par rapport à la façon de s'exprimer des habitants actuels? Les lexiques sontils comparables, par exemple pour désigner les lieux et leurs composantes paysagères? Se reconnaissent-ils dans les descriptions dressées par les auteurs et reconnaissent-ils leur monde ? Dévoiler les réseaux de l'intertextualité entre les deux modes de discours, repérer le dialogue sous-jacent qui leur permet d'exister : cela n'est réalisable qu'en croisant les deux types de discours.

Dans ces conditions, la question de la légitimité d'une comparaison entre discours de nature différente, que nous posions lors de la Journée des doctorants, n'apparait plus aussi pertinente. Comme l'a relevé Nathalie Blanc au cours des débats qui ont suivi, il ne s'agit peut-être pas de comparer des discours (ce qui induirait de poser face à face des éléments comparables, mais aussi de s'assurer que les deux sources de connaissance interfèrent suffisamment pour qu'il y ait matière à comparer) mais d'instaurer une forme de complémentarité entre les deux corpus avec pour unique souci d'échanger et d'instaurer un dialogue créateur de nouvelles représentations. La question de la légitimité ne se pose finalement plus que dans le choix des discours à associer et dans la méthodologie du dialogue.

Les modalités du dialogue entre romans et récits ordinaires

Le débat qui eut lieu lors de cette Journée a contribué à opérer ces choix et notamment à ne pas élire les discours de spécialistes : les rapports, les plans des professionnels qui interviennent sur les territoires urbains (architectes, urbanistes, paysagistes) certes construisent ou reconstruisent des mondes avec du langage; ces mondes sont cependant partiels (ils n'abordent qu'un projet ponctuel de plus ou moins grande échelle même s'il est inséré dans une étude préalable plus englobante) et leur langage «culturel » est plutôt iconographique, nous éloignant du caractère textuel sur lequel repose la méthode de lecture initiale. Il semble aussi indispensable que ces discours se déroulent dans un temps suffisamment long pour offrir les mêmes qualités d'analyse diachronique que proposent les romans insérés dans une œuvre, et qu'ils intègrent la possibilité de reformulations, de redondances, de contradictions. Toutes ces caractéristiques ne sont rassemblées que si l'on conserve la dimension subjective et donc individuelle de ceux qui s'expriment. Les habitants des quartiers mis en scène par les romans semblent tout désignés ${ }^{22}$. Reste à choisir le support pour les écouter: le choix entre matériau oral ou matériau écrit ne me semble pas essentiel, sinon que toute collecte de témoignages oraux permet un échange, un contact humain, qui est irremplaçable pour la propre expérience du chercheur. Par ailleurs, les écarts entre les 
deux modes d'expression oral et écrit, peuvent permettre de rendre compte de stratégies discursives différentes. Les paroles énoncées de manière continue exhibent par exemple des procédés de reformulation rarement perceptibles dans le matériau littéraire.

La méthode ethnosociologique propose une forme d'entretiens qui obéit à un certain nombre des postulats énoncés: ce sont les récits de $v^{23}{ }^{23}$. Ils permettent de maintenir le caractère narratif et subjectif des discours tout en étant le produit d'une interaction entre le locuteur et le récepteur (le sens du discours réside dans l'échange, de même que le sens d'un texte littéraire réside dans l'interaction lecteur / texte). La liberté de l'échange et le principe de coopération entre le chercheur et l'interlocuteur rendent possible l'émergence de formulations, thématiques, structures syntaxiques inattendues. Pour que le suivi des interlocuteurs soit aussi intense et proche que celui réalisé avec les quatre auteurs de romans, la construction d'une dizaine de biographies est suffisante au cours d'entretiens répétés sur plusieurs mois : il ne s'agit dans ce cas de répondre à aucune exigence de représentativité. Cette démarche ne cherche pas tant à vérifier des hypothèses par l'expérimentation (démarche hypothético-déductive) qu'à saisir une réalité et à élaborer des hypothèses tout au long de l'étude. Parallèlement aux discussions enregistrées, un travail d'ethnographe antérieur, simultané et postérieur aux entretiens est réalisé : il s'agit d'accumuler de l'information sur le quartier, la ville, ses habitants, leurs pratiques dans le quartier... et pendant les entretiens de noter le contexte d'énonciation, les détails qui semblent signifiants, dans les attitudes, les gestes, ce qui se passe autour, etc.

Bien évidemment il nous faut adapter la méthode à la démarche géographique et à celle de l'étude de paysage. On peut supposer qu'en retraçant leur vie, plus particulièrement mise en relation avec un quartier ou une ville, les habitants énonceront des descriptions paysagères, exprimeront leur relation à l'environnement (ce qui est la définition adoptée pour la notion de paysage) de façon spontanée. Cela n'exclut pas que le discours puisse être orienté dans ce sens par le chercheur soit par des questions, des demandes expresses, ou en proposant de réaliser des parcours ${ }^{24}$. Il est aussi demandé de dessiner sur des cartes un certain nombre de renseignements comme les limites du quartier ou celle du territoire personnel.

Pour favoriser le croisement de discours dans les deux sens, plusieurs projets de rencontre avec les romans peuvent être envisagés. Tout d'abord il peut s'agir de faire appel à la culture propre de l'interlocuteur, à ses lectures antérieures : connait-il des romans (ou tout autre forme de production littéraire) qui mettent en scène son quartier ou la ville de Barcelone? Parmi les œuvres de fiction qu'il connait (films, bandes dessinées etc.), qu'est-ce qui a le mieux montré la ville d'après lui ? Je lui demande ensuite d'en choisir un ou deux, de me raconter rapidement de quoi il s'agit, et de m'expliquer ce qui lui a plu dans cette œuvre. Le commentaire est peu à peu orienté vers la thématique du quartier ou de la ville par des questions telles que: "Comment parle-t-on du quartier dans ce roman? Par quoi est-il caractérisé ? Quelle est l'image dont tu te souviens le mieux (ce peut être une image, une représentation, un paysage du quartier ou de n'importe quel autre lieu)?». Pour le prochain entretien, je propose qu'il choisisse un extrait de ce roman en relation avec le quartier ou avec Barcelone afin de le commenter ensemble.

La seconde modalité consiste à ce que je choisisse des extraits parmi les romans qui ont été étudiés. Après l'avoir lu ensemble, une discussion générale s'engage et je cherche à 
savoir si l'interlocuteur se reconnaît dans ce qui été lu, s'il est d'accord avec ce qui est dit, si cela correspond à son image des lieux. Puis par des questions plus précises, de courts passages sont explicités et commentés. Les questions formulées sont de ce type : que signifie pour toi telle phrase ? Es-tu d'accord avec ce qui est écrit... ? Que penses-tu de cette description du quartier, de la rue... ? Il peut être demandé aussi des éclairages sur des aspects particuliers (fonction "informateur» de l'interlocuteur) ou au contraire on peut s'assurer qu'il connaissait telle ou telle chose.

Ces quelques exemples n'ont pour objectif que de préciser comment l'on peut introduire dans des entretiens le monde tel que la littérature l'exprime et quels sont les moyens simples qui permettent de faire surgir des discours originaux sur leur environnement. Bien d'autres "techniques " peuvent être expérimentées, il s'agit là encore de faire preuve d'un peu d'imagination.

La phase de recherche dans laquelle je suis actuellement est celle de la rencontre avec les habitants. Les entretiens sont réalisés et analysés au fur et à mesure, afin de faire évoluer les hypothèses et d'opérer un aller-retour constant avec la lecture des œuvres littéraires. Celles-ci ont déjà prouvé dans le cadre de cette recherche leur pertinence pour comprendre l'urbanité barcelonaise ; reste à valider l'hypothèse de la richesse du croisement de ces discours avec ceux des habitants. Nous ne pouvons donc pas produire de résultats à ce jour, mais seulement postuler que cette démarche vaut la peine d'être entreprise et généralisée.

\section{BIBLIOGRAPHIE}

Adam J.-M., Petitjean A., Revaz F. (1989) Le texte descriptif. Poétique historique et linguistique textuelle, Paris, Nathan, collection Nathan-Université, 239 p.

Bakhtine M. (1975) Esthétique et théorie du roman, 1978, Paris, NRF Gallimard, Bibliothèque des idées.

Berque A. (1990) Médiance de milieux en paysages, Géographiques-Reclus, 163 p.

Berque A., Conan M., Donadieu P., Lassus B., Roger A. (1999) Mouvance. Cinquante mots pour le paysage, Paris, Éditions de la Villette-Passage, 99 p.

Bertaux D. (1997) Les récits de vie. Perspective ethnosociologique, Paris, Nathan 128 p.

Brosseau M. (1989) Des romans géographes. Essai, Paris, L'Harmattan, Géographie et culture, 246 p.

Carreras i Verdaguer C. (1985) «La ciutat de Barcelona a les novelles de Joan Marsé », Revista Catalana de Geografia, Barcelona, no 1, desembre 1985, vol. I, p. 46-58.

Carreras C. (1988) « Paisaje urbano y novela », Estudios Geográficos, Madrid, tome XLIX, no 191, p. $165-187$.

Chevalier M. (2001) « Géographie et littérature, La Géographie ». Acta Geographica, Hors série no 1500 bis, Paris, Société de Géographie, 260 p. 
Compagnon A. (1998) Le démon de la théorie. Littérature et sens commun, Paris, Seuil, La couleur des idées, $311 \mathrm{p}$.

Díaz De Castro F., Quintana Peñuelas A. (1984) Juan Marsé: ciudad y novela, 'Últimas tardes con Teresa'; organización del espacio y producción de imagen, Palma de Mallorca, 95 p.

Fabre T. \& Ilbert R. (2000) Les représentations de la Méditerranée. Regards croisés sur la Méditerranée, Paris, Maisonneuve \& Larose, 10 volumes.

Goytisolo L. (1973) Recuento (Antagonía 1), Madrid, Alianza Editorial, El libro de bolsillo ed. 1987, $685 \mathrm{p}$.

Guillamon J. (2001) La ciutat interrompuda. De la contracultura a la Barcelona postolímpica, Barcelona, Edicions de la Magrana, $314 \mathrm{p}$.

Hamon Ph. (1988) Introduction à l'analyse descriptive, Paris, Hachette.

Lassave P. (2002) Sciences sociales et littérature, Paris, PUF, Sociologie d'aujourd'hui, 243 p.

Marsé J. (1966) Últimas tardes con Teresa, Barcelona, Plaza Janés, Biblioteca de Juan Marsé, sexta edición, 1998 ; trad. française de Saint-Lu J.-M. (1993) Teresa l'après-midi, Paris, Christian Bourgois éditeur, $433 \mathrm{p}$.

Marsé J. (1984) Ronda del Guinardó, Plaza Janés, Biblioteca de Juan Marsé, tercera edición 1998, Barcelona, trad. française de Saint-Lu J.-M. (1990) Boulevard du Guinardo, Paris, Christian Bourgois éditeur ; 1995, Paris, éditions 10/18 Christian Bourgois éditeur, collection Domaine étranger, 125 p.

Marsé J. (2000) Rabos de Lagartija, Barcelona, Editorial Lumen, (2002) Ave Fénix Debolsillo, Biblioteca Juan Marsé, 345 p. ; trad. française (2001) de Saint-Lu J.-M., Des lézards dans le ravin, Paris, Christian Bourgois éditeur, $404 \mathrm{p}$.

Mendoza E. (1975) La verdad sobre el caso Savolta, Barcelona, Seix Barral, biblioteca de Bolsillo, trigésima edición de 2001, 432 p., trad. française de Saint Lu J. M. (1986) La vérité sur l'affaire Savolta, Paris, Flammarion, Seuil, Points-roman, 1992.

Mendoza E. (1986) La ciudad de los prodigios, Barcelona, Seix Barral, biblioteca de Bolsillo, segunda edición en Biblioteca de Bolsillo, 475 p., trad. française d'Olivier Rolin, 1988, La ville des prodiges, Paris, éditions du Seuil.

Mendoza E. y C. (1989) Barcelona modernista, Barcelona, Planeta.

Mendoza E. (1996) Una comedia ligera, Barcelona, Seix Barral ; trad. de F. Maspéro, 1998, Une comédie légère, Seuil.

Montalbán M. V. (1990) Barcelonas, Barcelona, Empúries, trad. catalane de Xavier Lloveras, Barcelones, (1990), Barcelones, 335 p. ; trad. française de Georges Tyras, (2002), Ò, Paris, Seuil, 291 p.

Montalbán M. V. (1991) El laberinto griego, 1997, Barcelona, Planeta, serie Carvalho no 16, 189 p., trad. française de Bleton Cl., 1992, Le labyrinthe grec, Paris, Bourgois éditeur, coll. 10/18, 191 p.

Montalbán M. V. (1997) Le désir de mémoire. Entretien avec Georges Tyras, Vénissieux, Éditions Paroles d'aube, 285 p.

Merleau-Ponty M. (1945) Phénoménologie de la perception, Paris, Éditions Gallimard, réédité dans la collection Tel en 1976, édition de 1998, 531 p.

Merleau-Ponty M. (1964) L'Cil et l'Esprit, Paris, Éditions Gallimard, réédité dans la collection Folio essais en 1985, édition de 1990, 93 p. 
Mondada L. (2000) Décrire la ville. La construction des savoirs urbains dans l'interaction et dans le texte, Paris, Anthropos, collection Villes, 284 p.

Proust M. (1920) Le temps retrouvé, Paris, Gallimard collection Folio, édition de 1988, 442 p.

Roger A. (1978) Nus et paysages. Essai sur la fonction de l'art, Paris, Aubier Montaigne, Collection présence et pensée, $322 \mathrm{p}$.

Roger A. (1997) Court traité du paysage, Paris, NRF, Gallimard, Bibliothèque des Sciences Humaines, $199 \mathrm{p}$.

\section{NOTES}

1.Ma réflexion sur le croisement de discours littéraires et ordinaires a été initiée en premier lieu lors d'une intervention à la Journée des doctorants de l'UMR Ladyss (Cnrs, Paris I, Paris VIII, Paris X) organisée le 8 février 2002. Elle était intitulée : « Comparer des discours de nature différente pour étudier les paysages : quelle pertinence, quelle légitimité, quelle méthodologie?».

2.Cf. Proust M., Le temps retrouvé (p. 222 et sq.). Affilié à cette philosophie, Alain Roger a énoncé des hypothèses auxquelles se réfère ce travail : ce sont la fonction sociotranscendantale de l'art et le phénomène de l'artialisation paysagère ( $C f$. A. Roger, 1978 et 1997).

3.Cf. Merleau Ponty (1945 et 1964) : saisir le monde ("l'être au monde ») sans rompre la coappartenance du sensible et du sens passe par la parole conçue comme expression. Les pensées ne sont que paroles, les idées que matières linguistiques : le langage exprime "l'être-au-monde ". 4.Les informations de première main de cette " géographie sensible » sont à la fois fournies par le corps grâce aux cinq sens (la sensibilité comprise comme esthésie) et par l'affectivité concrétisée par des émotions et des sentiments présents ou sous forme de traces mémorielles (comme les souvenirs).

5.Le roman de Barcelone est une saga sans fin sur laquelle se penchent régulièrement les cercles littéraire, universitaire et culturel de la ville. Cf. par exemple les articles traitant de la Barcelone littéraire publiés à l'occasion des J.O. de 1992 ou l'ouvrage de J. Guillamon (2001). Par ailleurs, Barcelone est la capitale éditoriale de l'Espagne et de nombreux écrivains espagnols contemporains y vivent. Elle partage, avec Madrid, 80 \% de la production littéraire aujourd'hui.

6.Les Espagnols lisent peu en général bien que les publications soient très nombreuses. Luis Goytisolo a reçu le prix du Meilleur Roman décerné par un jury de critiques littéraires en 1985 et celui du ministère de la Culture en 1993.

7.Récompensé de nombreuses fois, lu et traduit dans le monde, il a obtenu le prix du Meilleur Livre Étranger à Paris en 1998 pour Una comedia ligera (1996) et l'ensemble de son œuvre.

8.Les écrivains espagnols contemporains entretiennent en général une relation serrée avec l'activité journalistique. Ils écrivent très régulièrement des colonnes dans les quotidiens et sont fréquemment issus du milieu journalistique.

9.Cette génération ancre son enfance dans la période des années de guerre civile et celles qui suivent (période du premier franquisme). Ils ont tous vécu activement la période dite de «transición » (passage du franquisme à la démocratie à partir de 1976 et pendant les années 80). 
10.Ce qui s'est traduit pour Mendoza et Montalbán par la réalisation d'ouvrages non fictionnels sur la ville. Cf. Cristina et E. Mendoza (1989) ; M. Vázquez Montalbán (1990). 11.Cette méthodologie s'appuie sur une tradition de dialogues théoriques et méthodologiques entre la littérature et les humanités, dont relève la géographie. $C f$. $P$. Lassave (2002) qui compare «l'évolution historique des rapports que chaque discipline [l'histoire, la sociologie, l'anthropologie ; la géographie étant exclue de sa réflexion] entretient avec la littérature » (p. 43) ; pour la géographie: M. Chevalier (2001), M. Brosseau (1989) et plus particulièrement sur le paysage Y. Luginbühl (1989) ou sur le paysage urbain Carles Carreras (1988). Sur la Barcelone littéraire (notamment Marsé), Cf. F. Díaz De Castro \& A. Quintana Peñuelas (1984), C. Carreras (1985). Ces études prennent peu en considération la dimension proprement fictionnelle et imaginaire des œuvres littéraires. Élaborer une méthodologie intégrant ces dimensions est une démarche relativement pionnière.

12. Cette étude systématique des composants et configurations paysagères récurrents a surtout été réalisée dans l'œuvre de Juan Marsé et de Montalbán. Chez Marsé par exemple, certains motifs ou configurations paysagères, inscrits dans une temporalité quasi-invariable de posguerra, endossent une valeur de signifié par métaphore, et représentent symboliquement le quartier de Gracia-Guinardó. À titre d'illustration, nous trouvons comme motifs le tramway 24 , les villas bourgeoises catalanes en cours de décomposition / déconstruction, les terrains vagues; la poussière rouge des collines que je commenterai plus loin ; les cerfs-volants élaborés par les enfants. Parmi les configurations composées, la place arborée animée avec fontaine ; les rues enchevêtrées, étroites, en pentes et souvent non goudronnées ; le chirrido des grillons, la végétation « sauvage ", l'aridité dans la « montagne », forment quelques-uns des éléments qui représentent le quartier.

13.Par exemple : « Derrière, la ville se serrait vers la mer sous une bande de ciel rose et gris. » La description du ciel indique ici la fin de la journée, au moment où le soleil disparaît. La composante paysagère a pour fonction d'indiquer un moment dans la narration, outre sa fonction poétique. Ronda del Guinardó (1984), trad. française, désormais RDG, p. 86.

14.Par exemple, le personnage de Lebrun dans le roman de Montalbán, El laberinto griego (1991), est défini par ce qu'il perçoit de la ville et ses réactions (ironie, fascination, enthousiasme, etc.).

15.Le sens attribué ici au terme de référent n'est pas celui classique de la linguistique mais celui qui le lie à la notion de mimesis, considérée comme reconstruction active de la réalité dans un récit afin de créer une réalité possible, acceptable pour le lecteur. (Ce sont donc à la fois les toponymes, les anthroponymes, les événements socio-

historiques... et le langage lui-même élaboré selon des codes sociaux dans le monde réel et au sein de la littérature). Cf. A. Compagnon (1998), chapitre « Le monde ».

16.Dans cet extrait (Marsé, RDG, p. 25) : « [...] au cours d'une nuit d'orage dans un terrain vague de la rue Cerdeña, quand elle avait été renversée près du feu de bois ; [...] », on reconnaît une scène de polar et toutes les connotations afférentes, mais aussi une rue réelle de Barcelone et un composant paysager réel dans les années50. Réel et fiction s'allient pour faire apparaître un paysage.

17.Cf. P. Lassave (2002, «Introduction : Littérature et sciences sociales : faux problème ou question vive?»). 
18.Cf. M.-A. Roque (2001) p. 26-29. L'étude a été menée par l'Institut Català de la Mediterrània auprès de 1200 leaders d'opinion, experts internationaux et institutions de la Méditerranée.

19.À cet égard le travail réalisé par dix chercheurs et dix écrivains de dix pays d'Europe sur les représentations de la Méditerranée, est très intéressant, notamment dans leur perspective « d'éclairage croisé » qui se matérialise par un texte fictionnel et un texte scientifique mis en regard pour chaque pays. Cf. T. Fabre \& R. Ilbert (2000).

20.Il s'agit de l'une des multiples descriptions du ravin qui constitue le cœur du roman (p. 86-87 de la traduction française).

21.Cf. M. Bakhtine (1975).

22.Nous entendons par habitants ceux qui investissent des lieux avec leur corps en y demeurant régulièrement, leur attribuant ainsi une charge symbolique qui leur permet de s'identifier à ce lieu au point de le nommer " mon territoire ». L'habitant d'une rue ou d'un quartier ne se confond évidemment pas avec le résidant ; de ce point de vue les écrivains (outre le fait qu'ils résident parfois dans les lieux qui inspirent leurs romans), qui errent sans fin dans les quartiers de la ville, habitent les lieux tout comme ceux qui les fréquentent régulièrement par plaisir, pour leur activité professionnelle ou autres. 23.La tradition, les postulats et les modalités de ce type d'entretiens sont clairement synthétisés par D. Bertaux (1997).

24.Ces parcours enregistrés constituent une forme d'adaptation de la méthodologie aux exigences de spatialisation de la géographie. Il est possible de proposer un parcours ou de se laisser guider sur le mode de la visite : dans ce cas on laisse l'interlocuteur aller où il veut, y compris en dehors du quartier prévu. Durant ces parcours, des notes peuvent être prises sur des cartes ou plans photocopiés, l'interlocuteur aura le loisir de produire des descriptions ou des commentaires en situation sur ce qu'il perçoit. Sur le site, j'envisage également de procéder à des lectures d'extraits de romans qui font ressortir des aspects particuliers d'un lieu et de demander de les commenter.

\section{RÉSUMÉS}

Afin de saisir comment réel et imaginaire se combinent pour construire l'identité de la ville de Barcelone, ou de ses lieux, l'auteur propose d'instaurer un dialogue entre des romans contemporains et des récits d'habitants barcelonais. Ces discours, compris comme traces de la relation habitants / ville (ce qu'elle nomme paysages) offrent une appréhension à la fois sensible et matérielle de la ville. Il s'agira de montrer en quoi les textes littéraires sont pertinents et nécessaires pour saisir les paysages barcelonais et pourquoi il est indispensable de croiser ces discours avec ceux recueillis auprès d'habitants ordinaires. Les modalités de ce dialogue seront définies et illustrées, dévoilant ainsi une pratique géographique métisse qui recourt à la sensibilité et à la rationalité et ne s'épanouit que par l'échange transdisciplinaire opéré entre littérature et sciences sociales.

In order to understand how reality and imagination combine to construct the identity of the city of Barcelona, I propose to create a link between contemporary novels and accounts from inhabitants of Barcelona. These testimonies are to be understood as traces of the relation 
between the dwellers and the city (what I call cityscape). They allow both a sensitive and a material understanding of the city. I will show how literary material is relevant and necessary to the understanding of the Barcelonian cityscapes and why it is essential to compare these accounts with those of ordinary citizens. The modality of this dialogue will be defined and exemplified, unveiling mixed geographical practices which appeal to sensitivity and rationality and develop only through a transdiciplinary exchange between literature and social sciences.

INDEX

Mots-clés : Ville

Index géographique : Espagne

\section{AUTEUR}

\section{SOPHIE SAVARY}

Agrégée de géographie, doctorante au Ladyss, université de Paris 1, allocataire de recherche, sophiesavary@freesurf.fr 